\title{
References
}

1. Kawerau, E., diese Z. 4, 224 (1966). - 2. Weller, C. and M. LINDER, Metabolism 10, 669 (1961). - 3. FerRarI, A., G. KESSLER, F. M. Russo-Alesi, J. M. Kelly, C. VANDeRwende and L. E. vaN Petren, Ann. New York Acad. Sci. 87, 729 (1960); 87, 745 (1960). - 4. Burns, T. W., R. Bregnant, H. J. van Peenan and T. E. Hood, Diabetes 14, 186 (1965). - 5. Galli, A., J. Jeanmaire, H. Chorsy and E. Schuller, Ann. Biol. clin. 19, 559 (1961). 6. Cästaigne, P., J. Cambier and E. Schuller, Technicon Symposium, Frankfurt (1965). - 7. Fitzgerald, G. and H. KEEN, Brit. Med. J., 1, 1568 (1964). - 6. HopfmaN, W. S., J. biol. Chemistry 120, 51 (1937). - 9. Kawerau, E., Technicon Symposium, p. 413, Frankfurt (1964). - 10. Field, J. B., H. E. Williams and G. E. Mortimore, J. Clin. Invest. 42, 497 (1963). 11. ZArowitz, H. and B. Ers, Ann. New York Acad. Sci. 74, 662 (1959). - 12. Creutzand, W., K. Wille and H. Kaup, Dtsch. med. Wschr. 87, 2189 (1962). - 13. UNGER, R. H. and L. L. Madrson, Diabetes 7, 455 (1958). - 14. JaCoBsen, A., Brit. Med. J., 1, 1507 (1962). - 15. Franckson, J. R. M., H.-A. Ooms, R. Bellens, V. Conrad and P. A. Bastenie, Metabolism 9, 482 (1962). - 16. Searle, G. L. and I. L. Chaikoff, Amer. J. Physiol. 170, 456 (1952). - 17. LundBAEK, K., Triangle 6, 194 (1964). -
18. Silverstone, F. A., M. Brandfonbrenee, N. W. Shock and M. J. Yiengst, J. Clin. Invest. 36, 504 (1957). - 18a. SilverStone, F. A., E. Soiomons and J. Rubricius, Diabetes 12, 398 (1963). - 19. Moorhouse, J. A., J. Steingerg and N. J. Rosen, Diabetes 12, 371 (1963). - 20. Stowers, J. M., P. D. Brewsher and R.G.BRACKenRIDGE, Diabetes 11 (Supplement), 127 (1962). 21. Lawrence, R. D., Med. Clin. North America 31, 289 (1947). 22. Marks, V. and D. Marrack, Clin. Sci., London 23, 103 (1962). - 23. Butterfield, W. J. H. and H. E. Holling, Clin. Sci., London 18, 147 (1959). - 42. Butrerfield, W. J. H., personal communication (1966). - 25. GUNDERSEN, K. and B. J. LiN, Diabetes 14, 805 (1965). - 26. Dolger, H., J. J. Bookman and C. Nechemas, Diabetes 11 (Supplement), 97 (1962). - 27. Pote, W.W.H. and R.L.Poucher, Diabetes 11 (Supplement), 132 (1962). - 28. West, K. M. and D. A. Wood, Amer. J. Med. Sc. 238, 25 (1959). - 29. Office of Health Economics Publication, No. 13 (1964). - 30. Turner, D. S. and N. McIntyre, Proc. Ass. Clin. Biochem. 3, 256 (1965). - 31. Arnoutd, Y., R. Bellens, J. R. M. Franckson and V. Conrad, Metabolism 12, 1122 (1963). - 32. SAmols, E. and V. MARKs, Lancet I, 462 (1965).
Dr. E. Kawerau, M. B., M. Sc., F. R. I. C. Department of Pathology, St. James Hospital, London, S.W.12/England

\section{Indirekte Bestimmung des Kohlenmonoxyds im Blut}

\author{
Von G. Ciuhandu, V. Rusu, M. Diaconovici und L. Kiss \\ Aus dem Laboratorium für Toxikologie des Institutes für Hygiene und Arbeitsscbutz, Timisoara, Rumänien
} (Direktor: Dr. E. Andriescu)

(Eingegangen am 14. Mai 1965)

\begin{abstract}
Eine früher ausgearbeitete Methode zur Bestimmung des ausgeatmeten Kohlenmonoxyds wurde verbessert.
Bei 80 Personen, die beruflich dem Kohlenmonoxyd exponiert waren, wurde gleichzeitig die Konzentration des Gases im Blute sowie das in 5 Min: bei Rückatmung von Sauerstoff ausgeschiedene Gas bestimmt. Die graphische Darstellung der erhaltenen Werte ergibt eine langsam steigende Kurve, die mit einer mittleren Genauigkeit von $\pm 0,5 \mathrm{ml}$ CO-proz. eine indirekte Bestimmung des $\mathrm{CO}-\mathrm{Hb}$ ermöglicht.

Bei einer 5 Min. währenden Rückatmung werden etwa $2 \%$ des gesamten, im Kreislauf befindlichen Kohlenmonoxyds ausgeschieden. Unter den beschriebenen Bedingungen wird ein Grenzwert von $2 \mathrm{ml}$ ausgeatmeten Kohlenmonoxyds als annehmbar vorgeschlagen.
\end{abstract}

Earlier methods for the determination of expired carbon monoxide have been improved. In 80 persons, who are exposed to $\mathrm{CO}$ in their occupations, the concentration of the gas was measured simultaneously in the blood and in the expired gas, following the reinspiration of oxygen. The resulting values show, graphically, a gradually increasing slope, form which the COHb level can be evaluated directly with a maximal dispersion of $\pm 0.5 \mathrm{ml}$ CO\%.

During $5 \mathrm{~min}$. reexpiration the sample contains about $2 \%$ of the total $\mathrm{CO}$ present in the circulation. Under the described conditions, a limit of $2 \mathrm{ml}$ of expired $\mathrm{CO}$ is suggested.

Die Kenntnis dẹs Gehaltes des Blutes an Kohlenmonoxyd in jedem beliebigen Moment einer gegebenen Zeit= periode - z. B. während der achtstündigen Arbeitszeit - ermöglicht die korrekte Deutung der unspezifischen Symptome, die bei Personen auftreten, die in einer CO-haltigen Atmosphäre arbeiten. Die bekannten, bei der Entnahme von signifikanten Blutproben auftretenden Schwierigkeiten ließen in letzter Zeit das Interesse an der Untersuchung des ausgeatmeten Gases wachsen. So schlug 1947 SHEPHERd (1) vor, die Analyse der ausgeatmeten Luft mit Hilfe eines MolybdatPalladiumsalz-Indikatorröhrchens durchzuführen. Ein
Jahr später versuchte SJöstrand (2) den CO-HbSpiegel indirekt dadurch zu bestimmen, daß er die CO- und $\mathrm{O}_{2}$-Konzentration in dem ausgeatmeten Gasgemisch nach Rückatmung von reinem Sauerstoff analysierte. - JoNEs und Mitarbeiter (3) gingen im Jahre 1958 den gleichen Weg und untersuchten das exhalierte Gas nach dem Einatmen von reinem Sauerstoff und darauffolgendem 20 Sek. langem Anhalten des Atems. Beide Verfahren wurden später in verschiedenen Modifikationen zur Erforschung des Kohlenmonoxyd-Sättigungszustandes des Organismus verwendet $(4-7)$. 
Die von Jones und Mitarbeitern (3) einerseits und von RINGOLD und Mitarbeitern (6) andererseits mit solchen Methoden erhaltenen Werte stimmen nicht überein. Bei den letzteren steigt der CO-Hb-Spiegel linear mit der Konzentration des ausgeatmeten Kohlenmonoxyds, bei den ersteren verläuft der Anstieg dagegen in Form einer Kurve. Die Differenzen sind mit der Veränderung der Sauerstoffkonzentration, mit möglichen Unterschieden im Restvolumen, im Sauerstoffverbrauch und in der unterschiedlichen Durchmischung der Gase zu erklären. HACKNEY und Mitarbeiter (5) lassen 2 Min. lang aus einem $4 l$-Sack Sauerstoff rückatmen und analysieren anschließend den Sackinhalt; diese Werte ergeben eine Kurve. Die zur Gleichgewichtseinstellung erforderliche Zeit kann hierbei bis zu 4 Min. betragen. Bei dieser Methode können auch Unterschiede im Ausgangsvolumen des Sacks stören.

Diese beiden Methoden erfordern stets die gleichzeitige Bestimmung von $\mathrm{CO}$ und $\mathrm{O}_{2}$ in der ausgeatmeten Probe und sind gegen Fehler, die durch die Änderung der Sauerstoffkonzentration im Gasgemisch auftreten, empfindlich. Wir versuchten diese Fehler dadurch auszuschalten, daß wir die gesamte Menge des im Laufe von 5 Min. ausgeschiedenen Gases nach Rückatmung von 10 l Sauerstoff bestimmten, wobei das $\mathrm{CO}_{2}$ gleichzeitig durch KOH-Lösung entfernt wurde. Wie wir in einer früheren Arbeit gezeigt haben (8) ist es möglich, das unter diesen Bedingungen ausgeschiedene $\mathrm{CO}$ - selbst bei beruflich nicht exponierten Nichtrauchern $(0,1$ bis $0,25 \mathrm{ml})$ - durch Farbreaktion mit alkalischer SilberSulfamoylbenzoesäure-Lösung ${ }^{1}$ ) photometrisch $\mathrm{zu}$ bestimmen. Die CO-Ausscheidung ist bei Rauchern 3 bis $6 \mathrm{mal}$ höher, erreicht jedoch bei beruflich exponierten Personen bis zu $5 \mathrm{ml}$.

In der vorliegenden Arbeit untersuchten wir, ob das durch Atmung ausgeschiedene Kohlenmonoxyd unter den oben geschilderten Bedingungen zur indirekten $\mathrm{Be}-$ stimmung dieses Gases im Blut dienen kann. $\mathrm{Zu}$ diesem Zweck wurden gleichzeitige Bestimmungen des ausgeatmeten und des im Blut enthaltenen Gases bei 80 in einem Hüttenwerk tätigen Personen durchgeführt. Die Bestimmung des Kohlenmonoxyds im Blute erfolgte dabei nach einem von uns ausgearbeiteten Verfahren (9) durch Diffusion des Gases in eine alkalische SilberSulfamoylbenzoesäure-Lösung.

\section{Methodik}

Die für die Rückatmung und Sammlung des ausgeatmeten Kohlenmonoxyds und seine Bestimmung erforderliche Apparatur wurde verbessert. Sie ist in Abbildung 1 wiedergegeben.

Das Spirometer besteht aus zwei Glaskörpern, wobei der untere - etwa $20 \mathrm{~m} l$ fassende $S_{1}$ - an einem Holzstativ befestigt und der obere - etwa $10 l$ Inhalt $S_{2}$ - beweglich an einer Zugrolle angebracht ist. Als Sperrflüssigkeit dient destilliertes Wasser. Das Gerät wird mit genau abgemessenen, durch die Öfnung $\mathrm{O}_{2}$ eingeführten Luftvolumina geeicht, wobei Klemme $b_{1}$ geschlossen ist. Die entsprechende Marke wird bei gewöhnlichem Luftdruck (Wasserspiegel in $V_{2}$ ) eingetragen.

1) Sulfamoylbenzoesäure $=$ p-Sulfamidbenzoesäure

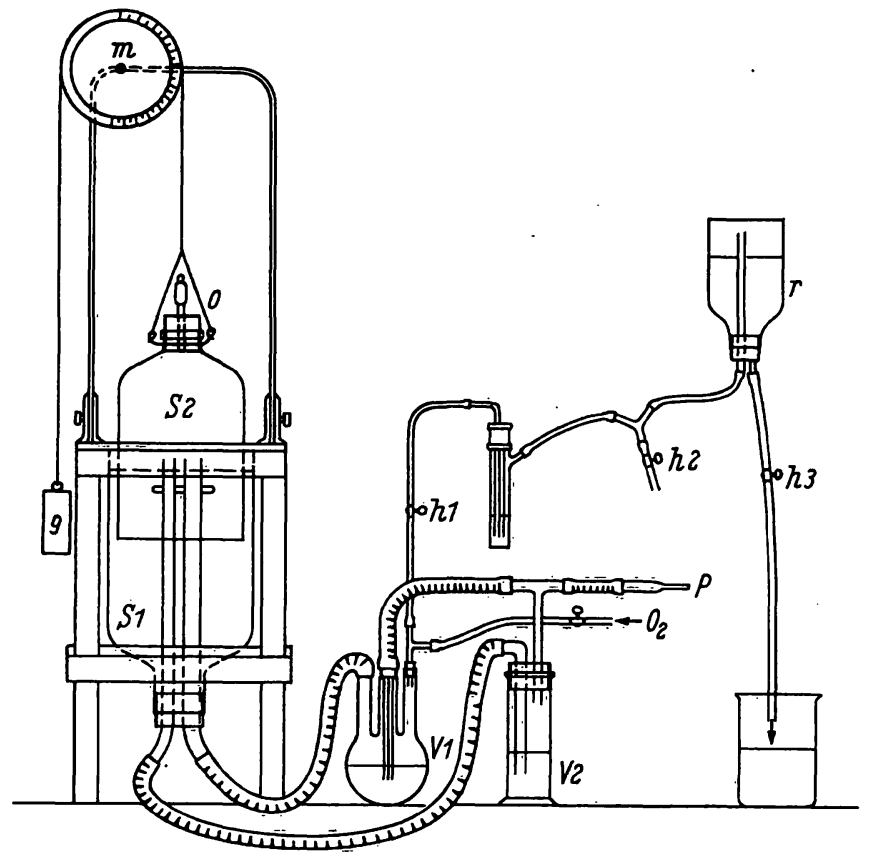

Abb. 1

Vor der Probenahme wird die obere Öfnung $O$ aufgemacht, der bewegliche Körper $S_{2}$ hinuntergedrückt und die Restluft mit Hilfe eines durch $\mathrm{O}_{2}$ eingeführten Sauerstoffstroms herausgetrieben. Man schließt $O$ und spült weiter mit Sauerstoff nach $P$. Der Körper $S_{2}$ wird freigelassen und hebt sich. Es werden $9 l$ Sauerstoff eingesaugt, welche mit dem Restgas zusammen $10 l$ ergeben. Das Volumen wird bei Luftdruck abgelesen (Wasserspiegel in $V_{2}$ ).

Die ausgeatmete Gasprobe wurde sofort nach der Blutentnahme durch das Glasmundstück $P$ unter $5 \mathrm{Min}$. lang währendem Einund Ausatmen erhalten. Die Restluft wurde vorher so weitgehend wie möglich aus der Lunge evakuiert. An der Zugrolle $\mathrm{m}$ wird das Volumen des unverbrauchten Sauerstoffs abgelesen (Wasserspiegel in $V_{2}$ ), wozu $1 /$ Restsauerstoff hinzugerechnet wird. Der Ausdruck „Restsauerstoff“ bezieht sich auf das in den Waschflaschen und Leitungen verbliebene Restvolumen Sauerstoff, dessen Größe als $1 l$ ermittelt wurde. Da sich das ausgeatmete CO auch in diesem mitverteilt, muß es entsprechend berücksichtigt werden. Nach etwa 10 Min. drückt man den beweglichen Glaskörper $S_{2}^{n}$ herunter und leitet den Sauerstoff durch eine Waschflasche mit 60-proz. Kalilauge $\left(V_{3}\right)$ in die Flasche $r$, die $1200 \mathrm{ml}$ faßt. Die Klemme $b_{2}$ wird zur Entfernung kleiner, aus der Flasche $r$ herrührender Wassermengen gelegentlich geöffnet. Nach Abfluß der letzten Wassertropfen werden in die umgedrehte Flasche $r$ durch das längere Rohr $10 \mathrm{~m} l$ Natrium-Silber-Sulfamoylbenzoatlösung gegeben.

Der Farbumschlag nach gelb erfolgt umso schneller, je höher die CO-Konzentration im Gasgemisch ist. Für eine näherungsweise Schnellbestimmung, vor allem bei akuten Vergiftungen, wird die Umschlagszeit nach Zugabe des Reagenzes gemessen, wobei eine $2 \cdot 10^{-4}$ molare Kaliumbichromatlösung als Vergleichsstandard dient (Abb. 2). Zur genauen Ermittlung des CO-Gehaltes wird nach $20 \mathrm{Stdn}$. Stehen bei $420 \mathrm{~m} \mu$ photometriert; Schichtdicke 1-20 mm. Die gesamte ausgeatmete Kohlenmonoxydmenge wird in der zuvor beschriebenen Weise berechnet (8). Die zur Aufstellung der Eichkurven benötigten $\mathrm{CO} / \mathrm{O}_{2}$-Gemische können durch ein geeignetes Verfahren leicht hergestellt werden (10).

\section{Ergebnisse}

Die Abhängigkeit des unter den oben beschriebenen $\mathrm{Be}-$ dingungen ausgeschiedenen Kohlenmonoxyds vom Gehalt des Blutes an diesem Gas wird durch eine Kurve ausgedrückt, deren Steigung mit der Gaskonzentration im Blute zunimmt. Dieser Kurvenverlauf scheint àuf 


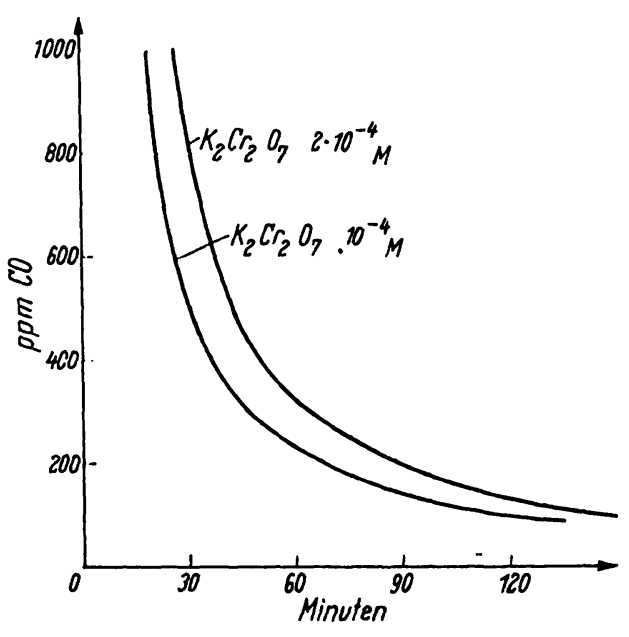

Abb. 2

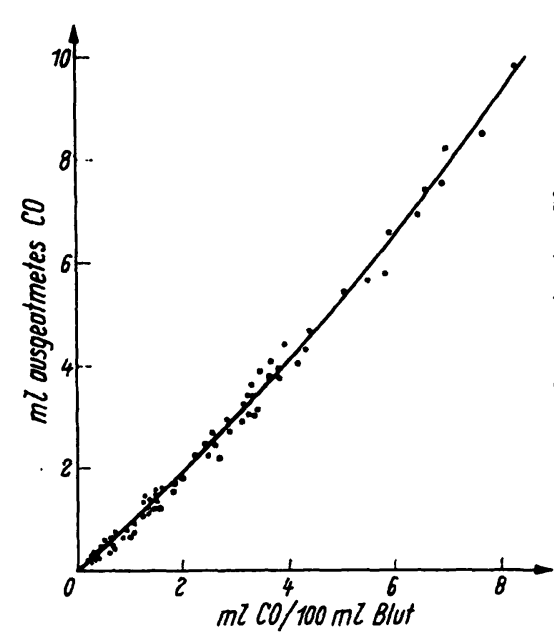

Abb. 3

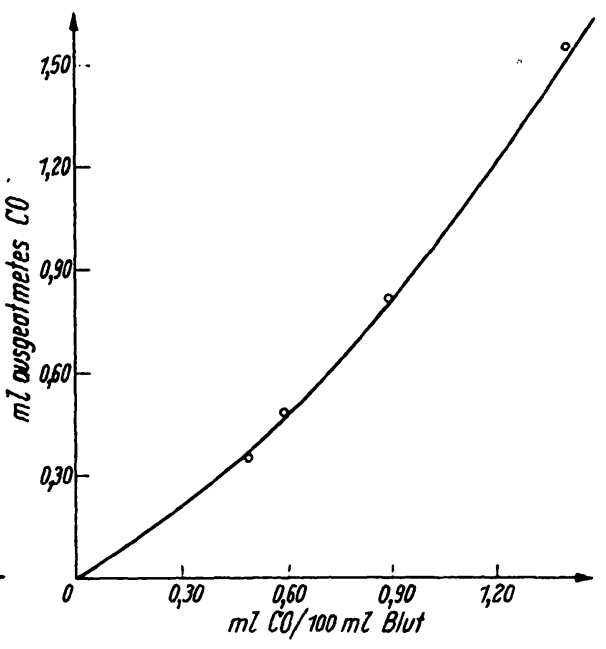

Abb. 4 eine Steigerung der Gasaustauschgeschwindigkeit bei höheren Kohlenmonoxydkonzentrationen hinzuweisen. Die Kurve ist in Abbildung 3 wiedergegeben.

Unsere Bestimmungen wurden an einer Gruppe ausgewählter Personen nach kurzer Unterweisung an Ort und Stelle ausgeführt. Die relativ geringe, unter $\pm 0,5 \mathrm{~m} l$ $\mathrm{CO} / 100 \mathrm{~m} l$ Blut liegende Streuung weist auf den begrenzten Einfluß individueller Faktoren hin. Die in Abbildung 3 wiedergegebene Kurve ermöglicht die indirekte Bestimmung des Kohlenmonoxyds im Blute bei einer ganzen Gruppe von Personen durch relativ einfache Analyse der ausgeatmeten Luft.

Bei ein und derselben Person erhielten wir für die Kohlenmonoxydausscheidung in Abhängigkeit von der Gaskonzentration im Blut eine übereinstimmende Kurve, wie Abbildung 4 verdeutlicht. Hier sind die Werte aufgetragen, die an einem der Autoren gemessen wurden, nachdem sich dieser freiwillig steigenden CO-Konzentrationen ausgesetzt hatte. Diese Kurve stimmt mit der in Abbildung 3 dargestellten gut überein. Beim Vergleich der in Abszisse und Ordinate aufgetragenen Werte fällt auf, daß das unter den von uns angegebenen Bedingungen ausgeatmete Kohlenmonoxyd-Volumen gröBenordnungsmäßig dem Gasgehalt in $100 \mathrm{ml}$ Blut ent- spricht. Nach Multiplikation mit 4 ergibt dieses Volumen annähernd den Prozentsatz an CO-Hb. Nimmt man als Mittelwert für das Gesamtvolumen $5 /$ Blut an, so ergibt sich, daß bei einer Probenahme etwa 2\% des gesamten im Kreislauf befindlichen Kohlenmonoxyds erfaßt werden. Damit ist eine gute Voraussetzung für die möglicherweise notwendige Wiederholung der Analyse gegeben.

Geht man davon aus, daß bei Gehalten von mehr als $2 \mathrm{~m} l \mathrm{CO} / 100 \mathrm{ml}$ Blut - entsprechend $8-10 \% \mathrm{CO}-$ $\mathrm{Hb}$ - bereits die klinischen Symptome einer Kohlenmonoxydvergiftung auftreten, so liegț unter den hier geschilderten Bedingungen der Probenahme der Grenzwert einer Intoxikation bei $2 \mathrm{~m} l$ ausgeatmetem CO. Unter diesen Grenzwert fällt auch die bei Rauchern vorkommende Ausscheidung.

Unspezifische Intoxikationserscheinungen - wie Kopfschmerz, Schwindel, Asthenie - die häufig mit einer erhöhten CO-Ausscheidung in Verbindung gebracht werden, lassen sich nunmehr gegen die bei CO-exponierten Personen auftretende Symptomatologie objektiv abgrenzen. Bei wiederholtem Auftreten dieser unspezifischen Symptome ist mit der Möglichkeit einer chronischen Intoxikation zu rechnen.

\section{Literatur}

1. SHEPERD, M., Ind. Engng. Chem. analyt. Edit. 19, 77 (1947). 2. SJöstrand, T., Acta physiol. scand. 16, 201 (1948). - 3. JonES, R. H., M. P. Elizicott, J. B. Cadigan und E. Gaensler, J. Laborat. Clin. Med., S. Louis 51, 553 (1958). - 4. Parmeggianr, L., S. Cambruzzi und G. Colombo, Med. lavoro, Milano 49, 428 (1958). - 5. Hackney, J. D., G. A. KaufmanN, H. Lashier und K. LxNN, Arch. environment. Health 5, 300 (1962). - 6. Ringord, A., P. Alto, J. R. Goldsmith, H. L. Hexwig, R. FinN und F.
Schuette, Arch. environment. Health 5, 308 (1962). - 7. GoldSMrth, J. R., J. Terzaghr und J. D. HACKNEY, Arch. environment. Health 7, 647 (1963). - 8. Ciuhandu, G., M. Diaconovici, L. KIss und V. Rusu, Hoppe-Seyler's Z. physiol. Chem., 339, 104 (1964). - 9. Crumandu, G. und V. Rusu, Z. analyt. Chem., im Druck. - 10. Cruhandu, G., V. Rusu und M. Diaconovicr, Z. analyt. Chem. 208, 81 (1965).

\section{Dr. G. Ciuhandu}

Timișoara (Rumänien), Str. Michelangelo 1 